\title{
On the mass and momentum transfer between short gravity waves and larger-scale motions
}

\author{
By K. HASSELMANN† \\ Institut für Geophysik, Universität Hamburg
}

(Received 16 September 1970 and in revised form 10 June 1971)

Interactions between short gravity waves and larger-scale flows are investigated in the two-scale approximation. The effect of the wave field on the mean flow is described by an interaction stress tensor and a surface mass transfer. The results are applied to Phillips' and Longuet-Higgins' model of short waves breaking on the crests of long carrier waves. It is found that the work done on the long waves by the interaction stresses (corresponding to Longuet-Higgins' 'maser' mechanism of wave generation) is almost exactly balanced by the loss of potential energy arising from the mass transfer. The residual energy transfer leads to attenuation of the long waves, independent of their propagation direction relative to the short waves. Damping factors are estimated from the upwinddownwind ratios of radar backscatter cross-sections. It is found that interactions with waves shorter than $35 \mathrm{~cm}$ yield attenuation rates about an order of magnitude smaller than the observed growth rates due to the wind.

\section{Introduction}

The interactions of short gravity waves propagating through a large-scale fluid motion have been the subject of a number of recent studies. Common to most approaches is some form of WKBJ approximation, in which the short waves are averaged over a space-time region large compared with the periodicities of the waves but small compared with the characteristic dimensions of the largescale (mean) flow.

The effect of the mean flow on the wave propagation can be derived elegantly by Whitham's (1967) averaged Lagrangian method (see also Bretherton \& Garrett 1968; Bisshopp 1969). For linear waves the main features follow from the conservation of wave action along wave-group trajectories. An alternative treatment has been given by Longuet-Higgins \& Stewart (1961).

The Lagrangian method can also be applied to determine the back interaction of the wave field on the mean flow. In this case the averaged Lagrangian density is varied with respect to the mean-flow parameters rather than the wave field. However the resulting Lagrangian equations are generally less convenient than the more usual Eulerian description. We shall accordingly derive the mean-flow equations here by the standard method of averaging the Eulerian equations of motion, together with the appropriate boundary conditions.

$\dagger$ Present addreas: Woods Hole Oceanographic Institution. 
The wave field is found to act on the mean flow in two ways. The usual Reynolds stresses can be combined with the mean pressure induced by the fluctuating wave velocities into an interaction stress tensor, which governs the transfer of momentum from the waves to the mean motion. Additionally, there is a transfer of mass due to the divergence of the Stokes mass transport. This term appears to have been overlooked in previous investigations.

The interaction stress is related to, but differs from, the radiation stress considered by Longuet-Higgins \& Stewart $(1960,1964)$. The radiation stress enters in the overall momentum balance of the complete flow consisting of the mean motion plus wave field. The interaction stress describes the transfer of momentum between the mean motion and the wave field. In simple cases, the net effect of both mass and momentum transfer can be deduced from the overall momentum balance and can therefore be expressed in terms of the radiation stress. However, in general the transfer processes need to be considered separately.

This is illustrated for the case of short waves interacting with long 'carrier' waves. It has been suggested by Phillips (1963) and Longuet-Higgins (1969a) that the hydrodynamic modulation of very short waves by longer carrier waves tends to cause breaking of the short waves on the long-wave crests. The subsequent regeneration of the short waves by the wind leads to a non-symmetrical distribution of the short-wave energy relative to the wave crests. Phillips investigated the back interaction of the resultant short-wave energy distribution on the long waves and concluded that the long waves would be attenuated. Recently Longuet-Higgins (1969a) has argued that the effect considered by Phillips is small compared with the energy transferred to the long waves during the white-capping process itself. A loss of momentum $\Delta \mathbf{M}$ of short waves breaking on the long-wave crests is assumed to correspond to an energy gain $\Delta E=\Delta \mathbf{M}$. $\mathbf{u}$ of the long waves, where $\mathbf{u}$ is the orbital velocity of the long waves at the crests. $\Delta E$ is positive if short and long waves are propagating in the same direction, and negative if the propagation directions are opposite. Thus in a growing sea the long waves would gain energy from the short waves, and it was suggested that this may be an important mechanism for wave generation.

It will be shown below that Longuet-Higgins' transfer term corresponds formally to the work done by the interaction stresses. However, a further energy transfer of opposite sign arises through the mass transfer. The modulation of the short waves by the long waves causes a divergence of the short-wave mass transport, which is balanced by a corresponding mass transfer to the long waves. Since mass is added and subtracted at different potential levels within the longwave cycle, this leads to a net transfer of potential energy. Thus if the short-wave mass transport is greater on the forward face of the long waves, mass of high potential energy is taken from the long-wave crests and is returned at a lower potential level in the wave troughs. To first order the potential energy lost in this way exactly balances the energy gained by the interaction stresses. The residual second-order transfer leads always to attenuation of the long waves, independent of their propagation direction relative to the short waves.

The attenuation factors can be determined experimentally from the quadrature component of the wave bispectrum. Unfortunately, in the normal fre- 
quency range of ocean wave records $(<1 \mathrm{c} / \mathrm{s})$, the quadrature bispectrum appears to be rather small and difficult to measure above the inherent statistical noise. However, measurements of radar return from the sea surface indicate a strong quadrature bispectrum at shorter wavelengths in the $\mathrm{cm}-\mathrm{dm}$ range. Estimates of the attenuation factors from the upwind-downwind ratio of backscatter crosssections yield $-35 \pm 10 \mathrm{db}$ per wave period - about an order of magnitude smaller than observed growth rates. However, this value should be regarded as a lower limit, since it excludes interactions with waves longer than $35 \mathrm{~cm}$ which lie beyond the conventional radar bands.

\section{Equations of motion of the mean flow}

The motion of an ideal homogeneous fluid of depth $h\left(x_{1}, x_{2}\right)$ with a free surface $x_{3}=\zeta\left(x_{1}, x_{2}, t\right)$ is governed by the equations

$$
\begin{array}{rlrlrl}
\frac{\partial u_{i}}{\partial t}+\frac{\partial}{\partial x_{j}}\left(u_{i} u_{j}\right)+\frac{\partial}{\partial x_{i}} p & =0, & -h \leqslant x_{3} \leqslant \zeta & & \text { (momentum equation), } \\
\partial u_{j} / \partial x_{j} & =0, & -h \leqslant x_{3} \leqslant \zeta & & \text { (incompressibility), } \\
p-g \zeta & =p^{a} & \text { at } \quad x_{3}=\zeta & & \text { (given pressure } p^{a} \text { at surface), } \\
\frac{\partial}{\partial t} \zeta+u_{\alpha} \frac{\partial \zeta}{\partial x_{\alpha}}-u_{3} & =0 & \text { at } & x_{3}=\zeta & & \text { (zero flow through surface), } \\
u_{\alpha} \partial h / \partial x_{\alpha}+u_{3} & =0 & \text { at } & x_{3}=-h & & \text { (zero flow through bottom), }
\end{array}
$$

together with appropriate conditions at the lateral boundaries. Latin indices run from 1 to 3, Greek indices from 1 to 2 , and $p$ denotes the kinematic pressure minus the equilibrium pressure $-g x_{3}$; the notation is otherwise standard.

We shall consider solutions consisting of a rapidly oscillating wave field $u_{i}^{\prime}, \zeta^{\prime}$ superposed on a mean flow $\bar{u}_{i}, \bar{\zeta}$ which varies slowly with respect to the horizontal co-ordinates and time. The mean properties of the wave field and the depth are also regarded as slowly varying functions. Means are defined in the usual manner as horizontal and time averages over dimensions intermediate between the periodicities of the wave field and the characteristic scales of the slowly varying fields.

The equations of motion of the mean flow can be derived by averaging (1)-(5), the interactions with the wave field arising through the non-linear terms and the pressure field. Applying the boundary conditions for the mean flow at the mean free surface $x_{3}=\bar{\zeta}$, the equations take the form

$$
\begin{aligned}
& \frac{\partial \bar{u}_{i}}{\partial t}+\frac{\partial}{\partial x_{j}} \bar{u}_{i} \bar{u}_{j}+\frac{\partial}{\partial x_{i}} p^{m}=\frac{\partial}{\partial x_{j}} T_{i j}^{\mathrm{int}}, \quad-h \leqslant x_{3} \leqslant \bar{\zeta}, \\
& \partial \bar{u}_{j} / \partial x_{j}=0, \quad-h \leqslant x_{3} \leqslant \bar{\zeta}, \\
& p^{m}-g \bar{\zeta}=\bar{p}^{a} \quad \text { at } \quad x_{3}=\bar{\zeta} \text {, } \\
& \frac{\partial \bar{\zeta}}{\partial t}+\bar{u}_{\alpha} \frac{\partial \bar{\zeta}}{\partial x_{\alpha}}-\bar{u}_{3}=-\frac{\partial}{\partial x_{\alpha}} M_{\alpha}^{w} \text { at } x_{3}=\bar{\zeta} \text {, } \\
& \bar{u}_{3}+\bar{u}_{\alpha} \partial h / \partial x_{\alpha}=0 \quad \text { at } \quad x_{3}=-h,
\end{aligned}
$$


where

$$
\left.T_{i j}^{\mathrm{int}}=-\overline{\left(u_{i}^{\prime} u_{j}^{\prime}\right.}+\delta_{i j} p^{w}\right)
$$

and

$$
M_{\alpha}^{w}=\int_{\bar{\zeta}}^{\bar{\zeta}+\zeta^{\prime}} u_{\alpha} d x_{3} .
$$

In averages such as (12) it is assumed (as in linear or weakly non-linear wave theory) that for $\zeta^{\prime}<0$ the fields can be continued analytically to the mean surface $x_{3}=\bar{\zeta}$.

The coupling with the wave field is expressed by two source terms: a force, given by the divergence of the interaction stress tensor $T_{i j}^{i n t}$, and a surface mass transfer due to the divergence of the wave mass transport $M_{\alpha}^{w}$.

The interaction stress consists of the Reynolds stress $-\overline{u_{i}^{\prime} u_{j}^{\prime}}$ and the contribution $p^{w}$ to the mean pressure field $\bar{p}=p^{m}+p^{w}$ arising from the wave field, the mean-flow pressure field $p^{m}$ being defined as the mean pressure in the absence of the wave field, i.e. the pressure field as determined from the usual Laplace equation and boundary conditions for the pressure with the fluctuating velocities and surface displacements set equal to zero. $\dagger$

The wave mass transport (12) represents the mean mass flow in the surface layer bounded by the instantaneous surface $x_{3}=\bar{\zeta}+\zeta^{\prime}$ and the mean surface $x_{3}=\bar{\zeta}$. The source term $-\partial M_{\alpha}^{w} / \partial x_{\alpha}$ in (9) follows by noting that according to (12)

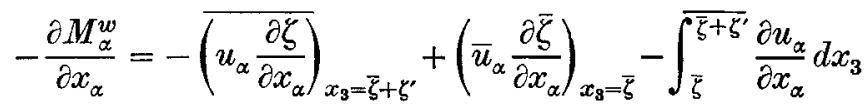

$$
\begin{aligned}
& =-\left\{u_{\alpha} \frac{\partial \zeta}{\partial x_{\alpha}}-u_{3}\right\}_{x_{3}=\bar{\zeta}+\zeta^{\circ}}+\left\{\bar{u}_{\alpha} \frac{\partial \bar{\zeta}}{\partial x_{\alpha}}-\bar{u}_{3}\right\}_{x_{3}=\bar{\zeta}},
\end{aligned}
$$

which is identical with the expression obtained by subtracting the left-hand sides of the mean flow boundary condition (9) and the mean of the boundary condition (4). Physically, condition (9) states that since there can be no mass flux through the instantaneous surface, the divergence $\partial M_{\alpha}^{w} / \partial x_{\alpha}$ of the horizontal mass flux $M_{\alpha}^{w}$ in the surface layer must be balanced by an equal flux upwards through the mean surface (cf. figure 1).

Since the wave mass transport in Eulerian co-ordinates is a surface phenomenon restricted to the region between the wave troughs and wave crests, the mass transfer affects the mean flow through a surface boundary condition (9). In the limit of infinitesimal surface displacements, the wave mass transport may be regarded formally as a surface current analogous to the electric surface current of an infinitely conducting medium. Conceptually, the Lagrangian description of the mass transport as a continuous particle drift extending about $\frac{1}{4}$ wavelength below the surface appears at first sight somewhat simpler. However, the momentum transfer is less easy to interpret in the Lagrangian reference frame. We have chosen a Eulerian approach here primarily to retain the accustomed interpretation of the momentum transfer in terms of mean Reynolds stresses and pressures acting on fixed surfaces.

$\dagger$ Thus equation (8) is not obtained by averaging (3), but by definition of the boundary condition for $p^{m}$. The difference between (8) and the average of (3) then yields a boundary condition for $p^{w}$. 
There is also a formal reason. We wish to distinguish between the mean flow and the wave field solely by averaging. In an entirely Lagrangian picture the wave mass transport would then appear as part of the mean flow, rather than a wave phenomenon. The wave-induced term is normally identified by referring also to Eulerian means: the Stokes drift is defined as the difference between the mean Lagrangian and the mean Eulerian current. Thus in both Lagrangian and Eulerian descriptions of the wave mass transport, the reference mean flow is defined in the Eulerian sense. For this reason it appears simpler to work entirely in the Eulerian frame, where the wave and mean-flow transports automatically separate into surface and interior contributions.

Mass balance

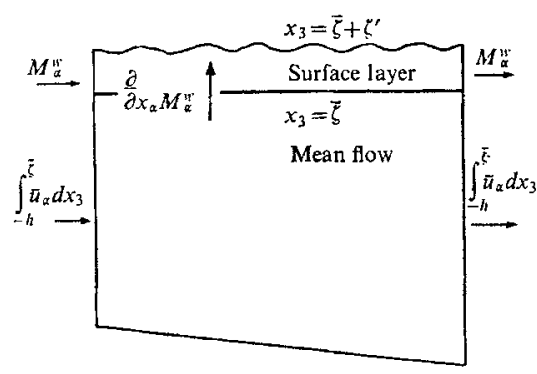

Momentum balance

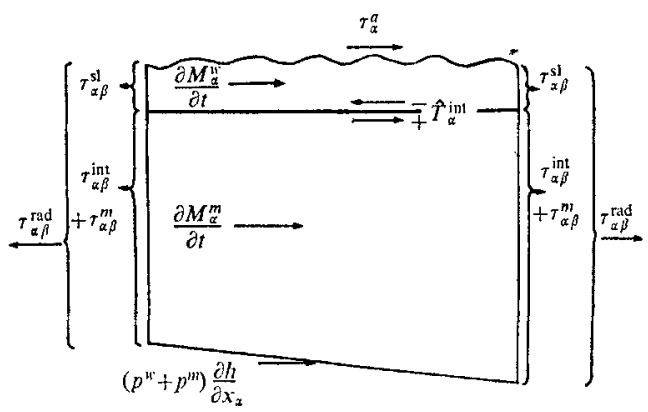

Frgure 1. Mass and momentum transfer between waves and mean motion. The wave momentum (Stokes mass transport) is concentrated in the surface layer between $x_{3}=\bar{\zeta}$ and $x_{3}=\bar{\zeta}+\zeta^{\prime}$. For better representation, the mean surface has been drawn below its true position through the centre of the waves.

We note that although we have referred to the field $\mathbf{u}^{\prime}, \zeta^{\prime}$ throughout as the 'wave' field, we have up to this point made no assumption regarding the dynamics of the fluctuating field. We have also nowhere invoked expansions of the boundary conditions with respect to $\zeta^{\prime}$ about the mean surface $x_{3}=\bar{\zeta}$ (except in the rather weak assumption of unique continuation of the fields for $\zeta^{\prime}<0$ up to the mean surface $x_{3}=\bar{\zeta}$ ). Thus (6)-(12) apply equally well to interactions with turbulence, or with a wave field which is modified by strongly non-linear dissipative processes such as white-capping.

The equations are not closed. The dependence of the interaction stress tensor and mass transfer on the mean flow is governed by the dynamics of the field $\mathbf{u}^{\prime}, \zeta^{\prime}$, which must be described by further equations. It is well known from theories of weak and strong interactions that a rigorously closed set of equations can be derived for the complete system only if the fluctuating field is an approximately linear wave field. However, we shall not be concerned in the following with the evaluation of the complete coupled system, but only with the back interaction of a given fluctuating field on the mean flow. In view of later applications to dissipative white-capping waves, it is important to recognize that (6)-(12) are not limited in this case to quasi-linear waves.

If the fluctuating field is, in fact, approximately linear, the expressions (11), (12) for the interaction stress and the mass transfer can be reduced to simple 
quadratic forms. Expanding the surface boundary condition for the pressure with respect to $\zeta^{\prime}$, the wave-induced mean pressure is readily seen to be $p^{w}:=-\overline{u_{3}^{\prime 2}}$ (Longuet-Higgins \& Stewart 1964), in accordance with the well-known expression for turbulent boundary-layers without a free surface. Hence

$$
T_{i j}^{\mathrm{int}}=\overline{u_{3}^{\prime 2}} \delta_{i j}-\overline{u_{i}^{\prime} u_{j}^{\prime}}
$$

Similarly, the expansion of (12) with respect to $\zeta^{\prime}$ yields

$$
M_{\alpha}^{w}=\left\{\overline{u_{\alpha}^{\prime} \zeta^{\prime}}+\frac{\partial \bar{u}_{\alpha}}{\partial x_{3}} \bar{\zeta}^{\prime 2}\right\}_{x_{\mathbf{3}}=\bar{\zeta}} .
$$

For an undamped deep-water gravity wave $\zeta^{\prime} \sim \cos (\mathbf{k} . \mathbf{x}-\omega t)$ the relations become

$$
\begin{aligned}
& T_{\alpha \beta}^{\mathrm{int}}=\omega^{2} e^{2 k x_{3}} \overline{\zeta^{\prime 2}}\left(\delta_{\alpha \beta}-k_{\alpha} k_{\beta} / k^{2}\right) \\
& T_{i 3}^{\mathrm{int}}=0, \\
& M_{\alpha}^{w}=\left(\omega k_{\alpha} / k\right) \overline{\zeta^{\prime 2}} .
\end{aligned}
$$

Thus in principal co-ordinates the interaction stress tensor reduces to a single horizontal component $\omega^{2} e^{2 k x_{s}} \overline{\zeta^{\prime 2}}$ parallel to the wave crests. If the wave is dissipative, additional components appear, including in particular the vertical shear stress terms $T_{\alpha 3}^{\mathrm{int}}$. A rotation of the system introduces further vertical components (Hasselmann 1970) but these are not relevant for the applications considered here.

\section{The vertically integrated momentum balance}

Previous investigations of wave-mean flow interactions have often been based on the concept of the radiation stress (Longuet-Higgins \& Stewart 1960, 1964). The distinction between the interaction stress and the radiation stress is best seen by considering the vertically integrated momentum balance of $(a)$ the mean flow, $(b)$ the complete flow and $(c)$ the surface layer between $x_{3}=\bar{\zeta}$ and $x_{3}=\bar{\zeta}+\zeta$ ' containing the 'wave' mass transport. The three equations are not independent, since the momentum $M_{\alpha}^{w}$ of the surface layer is given by the difference between the mean momentum $\bar{M}_{\alpha}$ of the complete field and the momentum $M_{\alpha}^{m}$ of the mean flow,

$$
\bar{M}_{\alpha}=\overline{\int_{-h}^{\zeta}} u_{\alpha} d x_{3}=\int_{-h}^{\bar{\zeta}} \bar{u}_{\alpha} d x_{3}+\overline{\int_{\bar{\zeta}}^{\bar{\zeta}+\bar{\zeta}^{\prime}}} u_{\alpha} d x_{3}=M_{\alpha}^{m}+M_{\alpha}^{w} .
$$

The rate of change of the mean-flow momentum follows by integrating the horizontal mean-flow equations of motion (6) from $-h$ to $\bar{\zeta}$. After some rearranging using the boundary conditions, the equation may be expressed in the form (which can also be obtained directly by considering the mean-flow momentum balance of the wedge in figure 1 )

$$
\frac{\partial M_{\alpha}^{m}}{\partial t}=\left\{\frac{\partial \tau_{\alpha \beta}^{m}}{\partial x_{\beta}}+\overline{p^{a}} \frac{\partial \bar{\zeta}}{\partial x_{3}}+\frac{\partial h}{\partial x_{\alpha}} p_{-h}^{m}\right\}+\frac{\partial \tau_{\alpha \beta}^{\mathrm{int}}}{\partial x_{\beta}}+\widehat{T}_{\alpha}^{\mathrm{int}}+\frac{\partial h}{\partial x_{\alpha}} p_{-h}^{w},
$$


where

$$
\begin{aligned}
\tau_{\alpha \beta}^{m} & =-\int_{-h}^{\zeta}\left(\bar{u}_{\alpha} \bar{u}_{\beta}+\delta_{\alpha \beta}\left(p^{m}-g x_{3}\right)\right) d x_{3}, \\
\tau_{\alpha \beta}^{\text {int }} & =\int_{-h}^{\bar{\zeta}} T_{\alpha \beta}^{\text {int }} d x_{3}, \\
\hat{T}_{\alpha}^{\text {int }} & =\left\{T_{\alpha 3}^{\text {int }}-\frac{\partial \bar{\zeta}}{\partial x_{\beta}} T_{\alpha \beta}^{\text {int }}-\frac{\partial M_{\beta}^{w}}{\partial x_{\beta}} \bar{u}_{\beta}\right\}_{x_{\alpha}=\bar{\zeta}} .
\end{aligned}
$$

and

The first parentheses on the right-hand side contain the usual momentum flux and pressure terms of the mean flow itself. The remaining terms arise from interactions with the fluctuating field; they consist of surface, bottom, and vertically integrated interaction stresses and an additional momentum transfer term due to the mass influx from the surface layer. (The interaction stress at the bottom reduces to the wave-pressure term $\left(\partial h / \partial x_{\alpha}\right) p_{-h}^{w}$, since the Reynolds stress contribution for an ideal fluid vanishes on account of the zero momentum convection through the bottom.)

To obtain the rate of change of the mean total momentum, we integrate first the horizontal equations of motion of the complete flow and then take averages. Making use of the boundary conditions, the result readily reduces to the form (which can again be inferred directly from figure 1)

$$
\frac{\partial \bar{M}_{\alpha}}{\partial t}=\left\{\frac{\partial \tau_{\alpha \beta}^{m}}{\partial x_{\beta}}+\bar{p}^{a} \frac{\partial \bar{\zeta}}{\partial x_{\alpha}}+\frac{\partial h}{\partial x_{\alpha}} p_{-h}^{m}\right\}+\frac{\partial \tau_{\alpha \beta}^{\mathrm{rad}}}{\partial x_{\beta}}+T_{\alpha}^{a}+\frac{\partial h}{\partial x_{\alpha}} p_{-h}^{w},
$$

where $T_{\alpha}^{a}$ is the mean momentum transfer from the atmosphere associated with the fluctuating component of the atmospheric surface stress (including both surface pressure and shear stresses), and the radiation stress

$$
\tau_{\alpha \beta}^{\mathrm{rad}}=\tau_{\alpha \beta}^{\mathrm{int}}+\tau_{\alpha \beta}^{\mathrm{sl}}
$$

is defined as the sum of the interaction stress and the mean stress

acting on the surface layer.

$$
\tau_{\alpha \beta}^{\mathrm{sl}}=-\overline{\int_{\bar{\zeta}}^{\bar{\zeta}+\zeta^{\prime}}\left(p \delta_{\alpha \beta}+u_{\alpha} u_{\beta}\right) d x_{3}}
$$

Subtracting (14) from (15) we obtain the momentum balance of the 'wave" field or surface layer

$$
\frac{\partial M_{\alpha}^{w}}{\partial t}=\frac{\partial \tau_{\alpha \beta}^{\mathrm{sl}}}{\partial x_{\beta}}+T_{\alpha}^{a}-\widehat{T}_{\alpha}^{\mathrm{int}}
$$

For an approximately linear wave field, the surface-layer stress simplifies to

$$
\tau_{\alpha \beta}^{\mathrm{sl}}=-\frac{1}{2} g \bar{\zeta}^{\prime 2} \delta_{\alpha \beta} \text {. }
$$

In the case of a deep-water wave $\zeta^{\prime} \sim \cos (\mathbf{k} \cdot \mathbf{x}-\omega t)$, the stressest $\tau_{\alpha \beta}^{\mathrm{int}}$ and $\tau_{\alpha \beta}^{\mathrm{rad}}$ are then given by

$$
\begin{aligned}
& \tau_{\alpha \beta}^{\text {int }}=\frac{1}{2} g \overline{\zeta^{\prime 2}}\left(\delta_{\alpha \beta}-k_{\alpha} k_{\beta} / k^{2}\right), \\
& \tau_{\alpha \beta}^{\mathrm{rad}}=-\frac{1}{2} g \zeta^{\prime 2} k_{\alpha} k_{\beta} / k^{2} .
\end{aligned}
$$

$\dagger$ Stresses denoted by $\tau$ have the dimension of a surface tension (force per line segment) rather than a stress in the usual sense of force per area. All stresses are defined in accordance with the standard sign convection (as used for Reynolds stresses, etc.). Longuet-Higgins \& Stewart (1964) define the radiation stress with an opposite sign. 
The stresses are equal in magnitude but perpendicular, the interaction stress acting parallel to the wave crests, whereas the radiation stress acts parallel to the direction of wave propagation.

The relations between the various stresses occurring in the three momentum balance equations (14), (15) and (18) are shown in figure 1.

We note that the radiation stress $\tau_{\alpha \beta}^{\mathrm{rad}}$ enters only in the overall momentum balance of the waves plus mean flow. This has caused some difficulties in applying radiation stress arguments to interactions between wave fields and slowly varying mean flows. A divergence of the radiation stress implies a rate of change of the overall momentum of the flow, but says nothing about the partition of this momentum change between the mean flow and the wave field; this can be inferred only from the interaction stress - or indirectly from additional consideration of the dynamics of the small-scale motions.

For approximately linear monochromatic deep-water waves propagating in the $x_{1}$ direction, the momentum balance equation (18) for the wave field may be written

$$
\frac{\partial M_{11}^{w}}{\partial t}+\frac{\partial}{\partial x_{1}}\left(v M_{1}\right)=T_{1}^{a}-\hat{T}_{1}^{\mathrm{int}},
$$

where $v$ is the group velocity of the waves. Thus, for given atmospheric input $T_{1}^{a}$, the momentum transfer $\widehat{T}_{1}^{\text {int }}$, which enters as an important driving term in the mean-flow momentum equation (14), can be determined from the spatial and time derivatives of the wave momentum in (18a). The latter quantities can be determined directly from wave spectral measurements. We shall make use of this relation in $\S \S 4$ and 5 .

The vertically integrated momentum-balance equations are particularly useful when the mean flow can be treated in the linear shallow-water approximation. In this case, the equations can be solved simultaneously with the vertically integrated continuity equation

$$
\frac{\partial \bar{\zeta}}{\partial t}+\frac{\partial}{\partial x_{\alpha}} M_{\alpha}^{m}=-\frac{\partial}{\partial x_{\alpha}} M_{\alpha}^{w}
$$

and the hydrostatic pressure relation $p^{m}=g \bar{\zeta}$. Equation (21) follows from (9) by replacing $\left(\bar{u}_{3}\right)_{x_{3}=0}$ by

$$
-\partial\left(\int_{-h}^{0} \bar{u}_{\alpha} d x_{3}\right) / \partial x_{\alpha}
$$

where $x_{3}=0$ is the undisturbed free surface.

If the characteristic propagation velocities of the transfer terms are small compared with the shallow-water phase velocity $(g h)^{\frac{1}{2}}$, the mean-flow response is quasi-static, and the term $\partial \bar{\zeta} / \partial t$ in (21) may be neglected. If the flow is furthermore independent of $x_{2}$, continuity yields $M_{1}^{m}+M_{1}^{w}=\bar{M}_{1}=$ const. : the mass flow must be the same at each section. But this implies constant total vertically integrated momentum. Hence the radiation and atmospheric stresses occurring in the overall momentum balance (15) must be opposed by an equally large hydrostatic pressure gradient. (For small bottom slope, the wave-pressure term $\left(\partial h / \partial x_{\alpha}\right) p_{-h}^{w}$ acting at the bottom may be neglected, since $p^{w}=-\overline{u_{3}^{\prime 2}} \approx 0$ at $x_{3}=-h$ on account of the bottom boundary condition (5). Similarly, for small 
mean currents and surface slopes the terms $\partial \tau_{\alpha \beta} / \partial x_{\beta}$ and $\overline{p^{a}} \partial \bar{\zeta} / \partial x_{\alpha}$ may be neglected.) The corresponding surface slope is given by

$$
g h \frac{\partial \bar{\zeta}}{\partial x_{1}}=\frac{\partial \tau^{\mathrm{rad}}}{\partial x_{1}}+T_{1}^{a}
$$

In this particular case the solution (due to Longuet-Higgins \& Stewart 1964) is seen to depend only on the radiation stress acting on the complete system, and not on the interaction stress and mass transfer. This is a specific consequence of the quasi-static approximation. In general, if the inertial forces are nonnegligible, the mean flow can be determined only by simultaneous consideration of both the mass and the momentum transfer.

In the following, we shall consider a mean flow consisting of long gravity waves, for which the inertial and potential forces are comparable. After determining the energy transfer from the short waves to the mean flow in terms of the interaction stress and the mass transfer, the final expression will again be found to depend only on the radiation stress, and a simple interpretation can again be given in terms of the momentum balance of the overall flow. In this case, the argument is based on the particular circumstance that the mean flow is a linear free-wave solution.

\section{The energy transfer between short and long gravity waves}

Consider now a 'mean' flow consisting of a statistical ensemble of long gravity waves. Averages of a quantity $q$ over the long-wave ensemble will be denoted by $\langle q\rangle$; short-wave averages are denoted as before by $\bar{q}$. The complete system is assumed to be statistically homogeneous, $\partial\langle q\rangle / \partial x_{\alpha}=0$. We assume further that the mean-flow equations can be linearized with respect to the long-wave amplitudes, and that the depth is infinite.

We derive first from the mean-flow equations of motion and boundary conditions (6)-(10) the rate of change of the mean-flow kinetic energy

$$
E_{\mathrm{kin}}=\left\langle\int_{-\infty}^{\bar{\zeta}} \frac{1}{2} \bar{u}_{i} \bar{u}_{i} d x_{3}\right\rangle \simeq\left\langle\int_{-\infty}^{0} \frac{1}{2} \bar{u}_{i} \bar{u}_{i} d x_{3}\right\rangle
$$

and potential energy $E_{\text {pot }}=\frac{1}{2} g\left\langle\bar{\zeta}^{2}\right\rangle$ :

$$
\begin{aligned}
& \frac{\partial E_{\text {kin }}}{\partial t}=\left\langle T_{\alpha 3}^{\text {int }} \bar{u}_{\alpha}\right\rangle_{x_{3}=0}-g\left\langle\bar{\zeta} \bar{u}_{3}\right\rangle_{x_{3}=0}-\int_{-\infty}^{0}\left\langle T_{i j}^{\text {int }} \frac{\partial \bar{u}_{i}}{\partial x_{j}}\right\rangle d x_{3}, \\
& \frac{\partial E_{\text {pot }}}{\partial t}=-g\left\langle\bar{\zeta} \frac{\partial}{\partial x_{\alpha}} M_{\alpha}^{w}\right\rangle+g\left\langle\bar{\zeta} \bar{u}_{3}\right\rangle_{x_{3}=0} .
\end{aligned}
$$

Thus the rate of change of the total energy $E=E_{\mathrm{k} 1 \mathrm{n}}+E_{\mathrm{pot}}$ is given by

$$
\frac{\partial E}{\partial t}=\left\langle T_{\alpha 3}^{\mathrm{int}} \bar{u}_{\alpha}\right\rangle_{0}-\int_{-\infty}^{0}\left\langle T_{i j}^{\mathrm{int}} \frac{\partial \bar{u}_{i}}{\partial x_{j}}\right\rangle d x_{3}-g\left\langle\bar{\zeta} \frac{\partial}{\partial x_{\alpha}} M_{\alpha}^{w}\right\rangle .
$$

$\dagger$ Generally, it can be shown that the energy transferred to a quasi-stationary linear system by external forces correlated with the overall motion of the system is divided among the individual modes of the system in proportion to their contribution to the relevant correlation products. In other words, equations of the form (22) apply to each mode separately. This establishes that the energy change (22) does indeed refer to the long waves, and not to other mean motions such as low-frequency currents. 
The first term represents the work done by the interaction stress at the surface. Only part of this energy goes into the mean motion; the rest is transferred back into the short waves via the second 'dissipation' term. The third term represents the net potential energy gained by adding mass at the rate $-\partial M_{\alpha}^{w} / \partial x_{\alpha}$ at the variable potential level $\bar{\zeta}$.

If the short waves are approximately linear, the cross products on the righthand side of (22) can be determined by expansion of the non-linear hydrodynamic wave equations. To lowest order, it is found that the short-wave energy is modulated in phase with the long-wave surface displacement. This implies that all three mean products in (22) vanish. However, out-of-phase terms leading to an energy transfer occur at higher order, in analogy with the higher-order transfer due to resonant interactions (cf. Phillips 1960; Hasselmann 1962). $\dagger$

An alternative, non-conservative transfer mechanism has been suggested by Phillips (1963) and Longuet-Higgins (1969 a). Longuet-Higgins \& Stewart (1960) have shown that the convergence of the flow on the forward face of the long waves tends to contract and steepen short waves as they are carried up towards the long-wave crests. If the short waves are near saturation, they will therefore tend to break near the wave crests. The effect is enhanced by the downward acceleration at the wave crests, which increases the white-capping instability by reducing the local virtual gravity. The subsequent regeneration of the short waves by the wind then leads to a non-symmetrical distribution of the short-wave energy relative to the wave crests, with the maximum short-wave energy shifted to the forward face of the long waves.

For our purposes the origin of the asymmetry leading to an energy transfer is irrelevant. We regard the short-wave field as given, assuming only that it satisfies the momentum-balance equation in the general form (18) (which contains no restriction with respect to the short-wave dynamics).

The momentum balance of the short waves can be used now to relate the work done by the surface interaction stress to the potential energy transfer. Assuming that the momentum transfer $T_{\alpha}^{a}$ from the atmosphere is not modulated by the long waves, the mean product of (18) with $\left(\bar{u}_{\alpha}\right)_{0}$ yields

$$
\left\langle T_{\alpha 3}^{\operatorname{int}} \bar{u}_{\alpha}\right\rangle_{0}=\left\langle\frac{\partial \tau_{\alpha \beta}^{\mathrm{sI}}}{\partial x_{\beta}}\left(\bar{u}_{\alpha}\right)_{0}\right\rangle-\left\langle\frac{\partial M_{\alpha}^{w}}{\partial t}\left(\bar{u}_{\alpha}\right)_{0}\right\rangle,
$$

where the stress $\widehat{T}_{\alpha}^{\mathrm{int}}$ in (18) has been replaced by $\left(T_{\alpha 3}^{\mathrm{int}}\right)_{x_{3}=0}$ on account of the linearity of the long-wave field. Adding the potential energy transfer, we obtain

$$
\left\langle T_{\alpha 3}^{\operatorname{int}} \bar{u}_{\alpha}\right\rangle_{0}-g\left\langle\bar{\zeta} \frac{\partial}{\partial x_{\alpha}} M_{\alpha}^{w}\right\rangle=\left\langle\frac{\partial T_{\alpha \beta}^{\mathrm{sl}}}{\partial x_{\beta}}\left(\bar{u}_{\alpha}\right)_{0}\right\rangle+\left\langle M_{\alpha}^{w}\left(\frac{\partial \bar{u}_{\alpha}}{\partial t}+g \frac{\partial \bar{\zeta}}{\partial x_{\alpha}}\right)_{0}\right\rangle
$$

where the derivatives in the last term on the right-hand side have been interchanged by invoking homogeneity and quasi-stationarity. But for a linear wave

$\dagger$ The present WKBJ limit is nevertheless basically different from the two-timing limit used in the random-field theory of resonant interactions. The conditions for the two forms of asymptotic expansion are mutually exclusive, so that the theories cannot be matched in a .common region of validity (cf. Hasselmann \& Schieler 1970). 
field, $\left(\partial \bar{u}_{\alpha} / \partial t\right)_{0}+g \partial \bar{\zeta} / \partial x_{\alpha}=0$ (equations (1) and (3)). Hence the last term vanishes, and the energy equation (22) reduces to

$$
\frac{\partial E}{\partial t}=\left\langle\frac{\partial \tau_{\alpha \beta}^{\mathrm{sl}}}{\partial x_{\beta}}\left(\bar{u}_{\alpha}\right)_{0}\right\rangle-\int_{-\infty}^{0}\left\langle T_{i j}^{\mathrm{int}} \frac{\partial \bar{u}_{i}}{\partial x_{j}}\right\rangle d x_{3} .
$$

The residual terms on the right-hand side of (24) are small compared with either the work done by the surface interaction stresses or the potential energy transfer. The orders of magnitude of the various terms can be estimated from the expressions $(11 b),(12 b)$ and $(17 a)$ for a linear wave field,

$$
\begin{gathered}
\left\langle\frac{\partial \tau_{\alpha \beta}^{\mathrm{sl}}}{\partial x_{\beta}}\left(\bar{u}_{\alpha}\right)_{0}\right\rangle / g\left\langle\bar{\zeta} \frac{\partial}{\partial x_{\alpha}} M_{\alpha}^{w}\right\rangle=O\left(\frac{\omega_{L}}{\omega_{S}}\right) \ll 1 \\
\int_{-\infty}^{0}\left\langle T_{i j}^{\mathrm{int}} \frac{\partial \bar{u}_{i}}{\partial x_{j}}\right\rangle d x_{3} / g\left\langle\bar{\zeta} \frac{\partial}{\partial x_{\alpha}} M_{\alpha}^{w}\right\rangle=O\left(\frac{\omega_{L}}{\omega_{S}}\right) \ll 1,
\end{gathered}
$$

where the indices $S$ and $L$ refer to the short and long waves, respectively. Substituting (22) and (24), it follows that the potential energy transfer and the work done by the surface interaction stress are both of order $\omega_{S} / \omega_{L}$ larger than the dissipation integral. However to first order both terms cancel, the residual being of the same order as the dissipation.

The right-hand side of (24) can be further simplified. Since the interaction stress is confined to a thin surface layer of order $k_{s}^{-1}$ within which the long-wave orbital velocity is essentially constant, the factor $\partial \bar{u}_{i} / \partial x_{j}$ in the second term can be taken outside the integral. The contribution from the vertical shear stress components $T_{\alpha 3}^{\text {int }}$ is then seen to be of order $k_{L} / k_{S}=\left(\omega_{L} / \omega_{S}\right)^{2}$ smaller than $\left\langle T_{\alpha 3}^{\mathrm{int}}\left(\bar{u}_{\alpha}\right)_{0}\right\rangle=O\left(g\left\langle\bar{\zeta} \partial M_{\alpha}^{w} \mid \partial x_{\alpha}\right\rangle\right)$. Since the first term on the right-hand side of (24) is only of order $\omega_{L} / \omega_{S}$ smaller than $g\left\langle\bar{\zeta} \partial M_{\alpha}^{w} \mid \partial x_{\alpha}\right\rangle$, the vertical shear stress terms can be neglected. The vertical normal stress $T_{33}^{\mathrm{int}}$ is presumably also small compared with the horizontal terms $T_{\alpha 3}^{\text {int }}$, since for a linear (but dissipative) wave field it vanishes identically (equation $(11 b)$ ). The remaining horizontal components then combine with the surface-layer stress in the first term to yield the radiation stress (equation (16)), so that finally

$$
\frac{\partial E}{\partial t}=\left\langle\frac{\partial \tau_{\alpha \beta}^{\mathrm{rad}}}{\partial x_{\beta}}\left(\bar{u}_{\alpha}\right)_{0}\right\rangle .
$$

Equation (25) may be compared with Longuet-Higgins' (1969a) result. By regarding only the momentum transferred from the short waves to the long waves, Longuet-Higgins essentially considered the work done by the surface interaction stress, $\left\langle T_{\alpha 3}^{\operatorname{int}}\left(\bar{u}_{\alpha}\right)_{0}\right\rangle$. If short-wave momentum is lost at the wave crests, the term is positive if short and long waves propagate in the same direction, and negative if the propagation directions are opposite. But the term is almost exactly balan ced by the potential energy transfer (figure 2); the residual transfer (25) leads always to attenuation of the long waves, independent of the relative propagation directions of short and long waves. For example, in the simplest case of a quasi-linear monochromatic wave travelling parallel to the $x_{1}$ direction, the radiation stress $(20)$ reduces to the single component $\tau_{11}^{\mathrm{rad}}=-\frac{1}{2} g^{\frac{1}{\zeta^{\prime 2}}}$, which is 
independent of propagation direction. If the short-wave energy is greater on the forward face of the long waves, $\partial \tau_{11}^{\mathrm{rad}} / \partial x_{1}$ is then of opposite sign to $\left(\bar{u}_{1}\right)_{0}$ on the wave crests, and the mean product $\left\langle\left(\partial \tau_{\alpha \beta}^{\mathrm{rad}} / \partial x_{\beta}\right)\left(\bar{u}_{\alpha}\right)_{0}\right\rangle$ is negative.

The occurrence of the radiation stress in the final expression (25) is at first sight surprising, since the radiation stress enters only in the momentum balance of the overall flow and not the mean motion. However, a simple physical explanation is suggested by considering the response of the overall flow to a variable radiation stress. Since the radiation stress is concentrated near the surface, it generates an oscillatory boundary layer of variable displacement thickness. By definition of the 'mean overall flow' the boundary layer includes

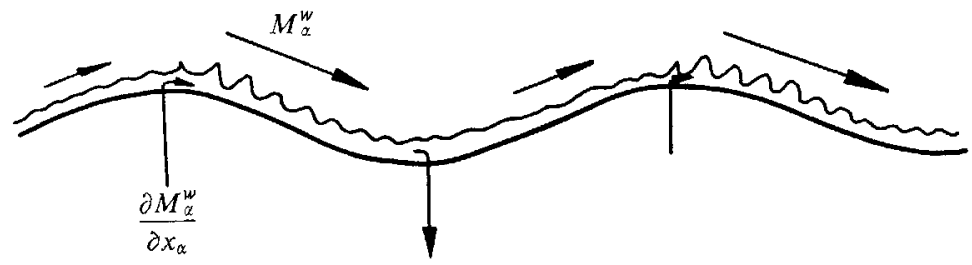

Figure 2. Mass transfer between long gravity waves and short modulated waves. Both waves are propagating from left to right. High-potential mass leaving the long waves at the crests is returned at lower potential in the troughs, resulting in a net extraction of potential energy from the long waves.

both the short-wave momentum in the 'infinitesimal' surface layer between $x_{3}=\bar{\zeta}$ and $x_{3}=\bar{\zeta}+\zeta^{\prime}$ and the mean-flow contribution from the finite surface layer below this (of about $\frac{1}{4}$ wavelength thickness) which is driven by the interaction stress. The variations in the displacement thickness lead to hydrostatic pressure variations beneath the boundary layer, which can then transfer energy to the mean motion by doing work against the mean vertical (long-wave) velocity.

The problem of a thin stress-driven boundary layer superposed on a free wave has been investigated in a related context by Longuet-Higgins $(1969 b) .+$ It was shown that the work done by the pressure forces against the vertical orbital velocity was equal to the work done by the shear stress against the horizontal orbital velocity - which is identical with the expression on the right-hand side of (25). The argument is based on the total boundary-layer variation and is therefore independent of the mass and momentum transfer between short and long waves. However, the equality of the work done by the shear stress and by the hydrostatic pressure applies only to a free-wave flow, so that the occurrence of the radiation stress in the energy balance of the mean flow is in a sense coincidental: although lengthier, the derivation in terms of the interaction stresses and mass transfer driving the mean motion yields perhaps a more direct physical description of the transfer process.

$\dagger$ See also Longuet-Higgins \& Stewart $(1964, \S 5)$. 


\section{Estimate of attenuation factors from radar sea clutter}

In the following, we assume that the radiation stress may be approximated by the relation (20) for a quasi-linear wave field. Applied to a spectrum $F(\mathbf{k})$ of short waves, where

the expression becomes

$$
\overline{\zeta^{\prime 2}}=\int F(\mathbf{k}) d \mathbf{k}
$$

Thus (25) may be written

$$
\tau_{\alpha \beta}^{\mathrm{rad}}=-\frac{1}{2} g \int F(\mathbf{k}) d \mathbf{k}
$$

$$
\frac{\partial E}{\partial t}=-\frac{1}{2} g \int\left\langle F(\mathbf{k}) \frac{\partial \bar{u}_{\alpha}}{\partial x_{\beta}}\right\rangle \frac{k_{\alpha} k_{\beta}}{k^{2}} d \mathbf{k} .
$$

The quantity $\left\langle\boldsymbol{F}(\mathbf{k}) \partial \bar{u}_{\alpha} / \partial x_{\beta}\right\rangle$ can be determined experimentally.

By resolving the long waves also into spectral components, the product can be expressed in terms of the quadrature bispectrum of the complete wave field. However attempts to measure the quadrature component of the bispectrum in the usual frequency range $(<1 \mathrm{c} / \mathrm{s})$ of ocean wave records appear to have been largely unsuccessful. Bispectral measurements by Hasselmann, Munk \& MacDonald (1963) agreed well with lowest-order non-linear hydrodynamic theory, which predicts a finite co-component and zero quadrature component. Barnett and Cartwright (private communications) both found no significant quadrature components in bispectra of wave-staff and buoy records.

However, at shorter wavelengths, evidence of a correlation between $F(\mathbf{k})$ and $\partial \bar{u}_{\alpha} / \partial x_{\beta}$ can be deduced from radar sea clutter measurements.

The general features of radar return from the sea surface can be well explained by lowest-order Bragg scattering theory. According to this model, an incident radar wave with wave-number $\mathbf{K}^{i}=\left(\mathbf{k}^{i}, k_{3}^{i}\right)$ is scattered by a gravity wave with wave-number $\mathbf{k}^{g}$ into two waves with wave-numbers $\mathbf{K}^{s}=\left(\mathbf{k}^{i} \pm \mathbf{k}^{g},-k_{3}^{i}\right)$. Thus there are two gravity waves $\mathbf{k}^{\theta}= \pm 2 \mathbf{k}^{i}$ which scatter into the back-scattered component $\mathbf{K}^{s}=-\mathbf{K}^{i}$, and the back-scattering cross-section per unit area of the sea surface is accordingly given by

$$
\sigma\left(\mathbf{K}^{i}\right)=T(\theta)\left[F\left(2 \mathbf{k}^{i}\right)+F\left(-2 \mathbf{k}^{i}\right)\right],
$$

where the transfer function $T(\theta)$ is a function of polarization and the depression angle $\theta$ relative to the horizontal. For electromagnetic waves, the ocean surface appears as essentially frozen. Thus the same transfer function occurs in both sum and difference interactions, independent of the sign of propagation of the scattering waves. The observed dependence of $T$ on polarization and $\theta$ has been shown by Wright (1968) to be in good agreement with theoretical calculations by Rice (1951) and Peake (1959).

However the lowest-order Bragg model has several short-comings. The observations indicate a significant cross-polarized return, which is not predicted by theory. The Doppler spectra are found to be appreciably broadened about the theoretical Bragg lines predicted at the Doppler frequencies $\omega^{g}= \pm(2 g k)^{\frac{1}{2}}$. And 
there is marked upwind-downwind asymmetry of the cross-section, in contradiction with the symmetrical form (27).

It has been suggested by Wright (1968) (see also Valenzuela 1968; Valenzuela \& Laing 1970; Hasselmann \& Schieler 1970) that these features can be explained by a 'composite-wave' model, in which the scattering gravity waves are superimposed on longer carrier waves. The long waves affect the local cross-sections through variation of the angles of incidence and polarization relative to the local scattering plane (electromagnetic interactions) and through modulation of the scattering wave spectrum (hydrodynamic interactions).

The cross-polarized return and the Doppler broadening can be explained qualitatively by the electromagnetic interactions. However, electromagnetic interactions do not affect the symmetry of the cross-section with respect to positive and negative wave propagation. Thus the observed upwind-downwind asymmetry must be of hydrodynamic origin. It will be shown that the asymmetry factor is closely related to the mean product occurring in equation (26).

If $p\left(n_{1}, n_{2}\right)$ represents the probability (horizontal density) distribution of longwave slopes $n_{\alpha}=\partial \bar{\zeta} / \partial x_{\alpha}$, the scattering cross-section in the composite-wave model is given by

$$
\sigma=\int \tilde{T}(\tilde{\theta})\left[\tilde{F}\left(2 \tilde{\mathbf{k}}^{i}\right)+\widetilde{F}\left(-2 \mathbf{k}^{i}\right)\right] p\left(n_{\mathbf{1}}, n_{2}\right) d n_{1} d n_{2}
$$

where the tilde denotes quantities defined with respect to the local long-wave facet. The function $T \rightarrow \widetilde{T}$ is affected both by the change of variables $\theta \rightarrow \tilde{\theta}$, $\mathbf{k}^{i} \rightarrow \tilde{\mathbf{k}}^{i}$ and by a rotation of the polarization axes. The change of the spectrum $F \rightarrow \widetilde{F}$ is due to hydrodynamic interactions. We assume that the hydrodynamical modulation $\Delta F$ is small,

$$
\tilde{F}\left(2 \tilde{\mathbf{k}}^{i}\right)=F\left(2 \tilde{\mathbf{k}}^{i}\right)+\Delta F\left(2 \tilde{\mathbf{k}}^{i}\right),
$$

where $\Delta F \ll F$ and $F$ denotes the unmodulated spectrum function.

For small-wave slopes, the functions $\widetilde{T}$ and $\widetilde{F}$ in (28) can be expanded in powers of $n_{\alpha}$. Since $\left\langle n_{\alpha}\right\rangle=\partial\langle\bar{\zeta}\rangle \mid \partial x_{\alpha}=0$, the leading terms are given by

$$
\sigma=\tilde{\sigma}_{0}+\frac{1}{2}\left(\frac{\partial^{2} \tilde{\sigma}}{\partial n_{\alpha} \partial n_{\beta}}\right)_{0}\left\langle n_{\alpha} n_{\beta}\right\rangle+\left(\frac{\partial \tilde{T}^{\prime}(\tilde{\theta})}{\partial n_{\alpha}}\right)_{0}\left\{\left\langle\Delta F^{\prime}\left(2 \mathbf{k}^{i}\right) n_{\alpha}\right\rangle+\left\langle\Delta F\left(-2 \mathbf{k}^{i}\right) n_{\alpha}\right\rangle\right\}+\ldots,
$$

where the subscript zero refers to values at $n_{\alpha}=0$ and

$$
\tilde{\sigma}=\tilde{T}(\tilde{\theta})\left[F\left(2 \tilde{\mathbf{k}}^{i}\right)+F\left(-2 \tilde{\mathbf{k}}^{i}\right)\right]
$$

represents the electrodynamic part of the modulated cross-section.

The first term on the right-hand side of (29) is the unmodulated cross-section as given by (27). The second term describes the lowest-order electromagnetic correction; since $\tilde{\sigma}$ is an even function of $n_{\alpha}$, it is symmetrical with respect to the incident angle. The third term arises from the hydrodynamical modulation, and is asymmetrical. The mean product $\left\langle\Delta F n_{\alpha}\right\rangle$ in this term is non-zero if the modulation of the spectrum is out of phase with the long-wave surface displacement-in accordance with the phase condition for an energy transfer between long and short waves. 
If, as assumed, the energy density of the short scattering waves is greater on the forward than on the rearward face of the long waves, the largest contribution to the scattering cross-section arises from rays incident on the forward face. For these rays, the effective depression angle is greater in the upwind than in the downwind direction. Since the transfer function increases with depression angle (for small depression angles), equation (29) predicts upwind-downwind crosssection ratios greater than one. This is in accordance with observations (cf. Marks 1965; Daley $1966 a, b)$. At larger depression angles $\partial T / \partial \theta$ decreases and becomes negative for vertical polarization (Wright 1968, figure 1). The trend to smaller upwind-downwind ratios with increasing $\theta$ is also confirmed by observations (within the considerable experimental scatter, cf. Daley (1966 , figure 6 and 7)).

With the aid of (29), the correlation between $\Delta F$ and the wave slope $n_{1}$ parallel to the wind can be expressed in terms of the upwind-downwind asymmetry

$$
\left\langle\Delta F\left(2 \mathbf{k}^{i}\right) n_{1}\right\rangle=-\left(\frac{\sigma_{u}}{\sigma_{d}}-1\right) \frac{F\left(2 \mathbf{k}^{i}\right) T}{2 \partial T / \partial \theta},
$$

where the wind is assumed to be blowing in the $x_{1}$ direction. (The term $\left\langle\Delta F n_{2}\right\rangle$ vanishes for reasons of symmetry.)

A reasonable mean value of Daley's measurements at the four radar wavelengths $3 \cdot 37,6 \cdot 75,24.5$ and $70 \mathrm{~cm}$ appears to be $\sigma_{u} / \sigma_{d} \approx 2$. Since the ratio tends to decrease rather rapidly betweed 24.5 and $70 \mathrm{~cm}$, we set $\sigma_{u} / \sigma_{d}=1$ for wavelengths longer than $70 \mathrm{~cm}$.

Within the approximations of the present estimate, the wave field may be treated for simplicity as unidirectional. The term $\partial \bar{u}_{\alpha} / \partial x_{\beta}$ in (26) can then be replaced by $\partial \bar{u}_{1} / \partial x_{1} \approx \bar{u}_{1} / \bar{\omega}$, where $\bar{\omega}$ is the mean frequency of the long waves.

At scattering wavelengths, the (one-dimensional) wave spectrum can be represented by Phillips' (1966, p. $109 \mathrm{ff}$.) saturation form $F(k)=\frac{1}{2} \alpha k^{-3}$, where $\alpha \approx 10^{-2}$. Substituting in (26), we obtain as attenuation rate of the long waves per unit wave period

$$
\beta=\frac{2 \pi}{\bar{\omega} E} \frac{\partial E}{\partial t}=-\frac{\alpha}{16} \frac{\lambda_{c}^{2}}{2 \pi} \frac{g}{\bar{E}} \frac{T}{\partial T / \partial \theta}\left(\frac{\sigma_{u}}{\sigma_{d}}-1\right),
$$

where $\lambda_{c}$ is the cut-off gravity wavelength,

$$
\lambda_{c} \approx 70 / 2=35 \mathrm{~cm} \text {. }
$$

At $\theta=15^{\circ}, T /(\partial T / \partial \theta)$ is of the order 0.6 for vertical polarization and 0.2 for horizontal polarization (Wright 1968). Taking a mean value of 0.4 and a meansquare amplitude $E / g=1 \mathrm{~m}^{2} / \mathrm{sec}$, equation (31) yields $\beta \approx-2 \cdot 10^{-4}$. This corresponds to a decay time of about half a day for a 10 sec wave.

The maximal growth rates in a wind-generated sea are at least an order of magnitude larger, $\beta_{\text {growth }} \approx 10^{-2}-10^{-3}$ (Snyder \& Cox 1966; Barnett \&Wilkerson 1967). A decay rate of $10^{-4}$ could be significant for swell propagation, but the measured upwind-downwind ratios probably apply only to wind waves, since the slopes of ocean swell are normally too small to contribute appreciably to the total 'long-wave' slope $\bar{n}_{\alpha}$. This is supported by the swell-attenuation measure- 
ments of Snodgrass et al. (1966), in which well dispersed swell was found to propagate over distances of many thousands of kilometres in the Pacific without measurable attenuation $\left(\left|\beta_{\text {swell }}\right|<10^{-5}\right)$.

We conclude tentatively that the energy loss of a wind sea due to interactions with waves shorter than $35 \mathrm{~cm}$ is of marginal significance. However, the expression (31) depends on parameters such as $\alpha$ which, for short waves, are only poorly known. The cut-off wavelength $\lambda_{c}$ is a particularly critical parameter. A relatively small upwind-downwind asymmetry at radar wavelengths longer than $70 \mathrm{~cm}$ could increase the estimate of $\beta$ considerably. Radar measurements in this range, if possible coupled with higher resolution wave measurements, would be of great interest in deciding whether radar return from the sea is a potentially useful tool for studying not only the 'state of the sea', but also wave dynamics.

This work was supported in part by the Office of Naval Research under contract N00014-69-0057.

\section{REFERENCES}

Barnett, T. P. \& Wilkerson, J. C. 1967 On the generation of wind waves as inferred from airborne radar measurements of fetch-limited spectra. J. Mar. Res. 25, 292-328.

Bisshopp, F.E. 1969 Nonlinear wave propagation in the geometrical approximation. Div. Appl. Math., Brown Univ. Tech. Rep. no. 9.

Bretherton, F. P. \& Garrett, C. J. R. 1968 Wave trains in inhomogeneous moving media. Proc. Roy. Soc. A 302, 529-554.

Daley, J. C. 1966 a Sea clutter measurements at $X$ and $C$ band. Nav. Res. Lab. Rep.

Daley, J.C. $1966 b$ Airborne radar backscatter study at four frequencies. Nav. Res. Lab. Rep.

HasselmanN, K. 1962 On the nonlinear energy transfer in a gravity-wave spectrum. Part 1. General theory. J. Fluid Mech. 12, 481-500.

Hasselmann, K. 1970 Wave-driven inertial oscillations. Geophys. Fluid Dyn. 1, 463-502.

Hasselmann, K., Munk, W. H. \& MacDonald, G. J. F. 1963 Bispectra of ocean waves. In Time Series Analysis (ed. M. Rosenblatt), ch. 8. Wiley.

Hassemanan, K. \& Schieler, M. 1970 Doppler spectra of electromagnetic backscatter from the sea surface at centimeter-decimeter and decameter wavelengths. Proc. Eighth Naval Hydrodyn. Conf., Pasadena (in the Press).

LongUet-Higgins, M. S. $1969 a$ A nonlinear mechanism for the generation of sea waves. Proc. R. Soc. A.311, 371-389.

LONGUET-HIacins, M. S. $1969 b$ Action of a variable stress at the surface of water waves. Phys. Fluids, 12, 737-740.

Longuet-Higgins, M. S. \& Stewart, R. W. 1960 Changes in the form of short gravity waves on long waves and tidal currents. J. Fluid Mech. 8, 565-583.

Lonfuet-Higgins, M. S. \& Stewart, R. W. 1961 The changes in amplitude of short gravity waves on steady non-uniform currents. J. Fluid Mech. 10, 529-549.

Longuet-Higgins, M. S. \& Sthewart, R. W. 1964 Radiation stresses in water waves; a physical discussion, with applications. Deep-Sea Res. 11, 529-562.

MARKs, W. 1965 The application of airborne radar backscatter to measurement of the state of sea. In Oceanography from Space (ed. G. Ewing), pp. 377-391. Woods Hole Oceanographic Inst.

Peake, W. H. 1959 Theory of rader return from Terrain. IRE Nat. Conv. Rec. 7, 27-41.

PHILlIPS, O.M. 1960 On the dynamics of unsteady gravity waves of finite amplitude. Part 1. The elementary interactions. J. Fluid Mech. 9, 193-217. 
PHILlirs, O. M. 1963 On the attenuation of long gravity waves by short breaking waves. J. Fluid Mech. 16, 321-332.

Phillips, O. M. 1966 The Dynamics of the Upper Ocean. Cambridge University Press.

RrCE, S. O. 1951 Reflection of electromagnetic waves from slightly rough surfaces. Comm. Pure Appl. Math. 4, 351-378.

Snodgrass, F. E., Groves, G. W., Hasselmann, K. F., Muller, G. R., Munk, W. H. \& Powers, W. H. 1966 Propagation of ocean swell across the Pacific. Phil. Trans. A 259, 431-497.

SNYDER, R. L. \& Cox, C. S. 1966 A field study of the wind generation of ocean waves. J. Mar. Res. 24, 141-178.

VALENZUELA, G. R. 1968 Scattering of electromagnetio waves from a tilted slightlyrough surface. Radio Science, 3, 1057-1066.

Valuenzumla, G. R. \& Laing, M. B. 1970 Study of Doppler spectra of radar sea echo. J. Geophys. Res. 75, 551-563.

WhiтнAM, G. B. 1967 Variational methods and applications to water waves. Proc. Roy. Soc. A 299, 6-25.

WRIGHT, J. W. 1968 A new model for sea clutter. IEEE Trans. AP-16, 217-223. 